\title{
Characterization of rectal, proximal and distal colon cancers based on clinicopathological, molecular and protein profiles
}

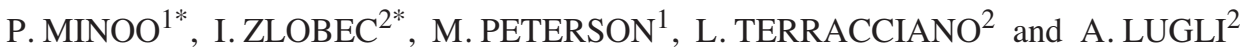 \\ ${ }^{1}$ Department of Pathology, University of California San Diego, San Diego, CA, USA; \\ ${ }^{2}$ Institute of Pathology, University Hospital of Basel, Basel, Switzerland
}

Received April 8, 2010; Accepted May 19, 2010

DOI: 10.3892/ijo_00000720

\begin{abstract}
Accumulating evidence suggests that colorectal cancer (CRC) should be viewed as a heterogeneous disease, with proximal and distal CRCs showing multiple biological and clinical differences. The aim of this study was to develop a clinicopathological, molecular and protein profile for CRCs based on their region and thus providing insight into their heterogeneity. CRC patients $(n=399)$ were evaluated for clinicopathologic and molecular features including $K-R A S$, $B R A F$ and MSI status. Tumors were also screened for expression of 50 immunohistochemical markers linked to major signaling pathways involved in tumor-progression or immune response. Proximally located tumors show significantly larger tumor size, higher T-stage, higher tumor grade and more frequent mucinous histologic subtype compared to the distal colon and rectum. The frequency of $B R A F$ mutation and MSI-high phenotype were significantly higher in proximal colon cancers. There is a significant difference in regional expression of 10 tumor-associated markers (CDX2, CD44v6, CD44s, TOPK, nuclear ß-catenin, pERK, APAF-1, E-cadherin, p21 and bcl2) and 4 immune response markers (CD68, CD163, FoxP3 and TIA-1). In multivariate analysis CD44s, CD44v6, nuclear B-catenin and CD68 expression was found to best discriminate left- versus right-sided colon cancers. Tumor diameter, pT stage and MSI status best distinguish right-sided colon cancers from rectal cancers and pT stage and E-cadherin best discriminate left-sided colon cancers and rectal cancers. These data along with existing evidence for the presence of distinct regional embryological origin and gene expression profile are highly supportive of the concept that proximal and distal CRCs are distinct clinicopathologic entities.
\end{abstract}

Correspondence to: Dr Parham Minoo, Department of Pathology, University of California San Diego, 200 West Arbor Dr. 8720, San Diego, CA 92103, USA

E-mail:pminoo@ucsd.edu

*Contributed equally

Key words: proximal colon cancer, distal colon cancer, rectal cancer, molecular profile, protein profile

\section{Introduction}

Colorectal cancer (CRC) is one of the leading causes of cancer mortality in North America and worldwide (1). Recent epidemiologic studies have reported a proximal migration of CRCs evidenced by increased incidence of right-sided colon cancers and a decrease in incidence of rectosigmoid tumors $(2,3)$, suggestive of different risk factors associated with carcinogenesis in proximal and distal colon $(4,5)$. These data support the concept originally proposed by Bufill in 1990 that cancers originating from these two different anatomical locations should have distinct molecular pathogenesis (6). Several biological differences between the normal proximal and distal colon (relative to the splenic flexure) may contribute to tumorigenesis in these locations along different pathways. For example, they have different embryonic origins: proximal colon derives from midgut while distal colon and rectum originates from embryonic hindgut. In addition, adult mucosal epithelium of proximal and distal colon can be distinguished by their pattern of gene expression since $>1,000$ genes are expressed differentially in adult ascending versus descending colon (7). Physiologically, sodium and water absorption commonly take place in proximal colon and progressively decreased toward rectum where fecal storage comprises the main physiologic function. Bacterial fermentation produces short chain fatty acids predominantly in proximal colon where they can be passively absorbed $(4,5,8)$. Furthermore, biliary salts shown to be associated with increased risk of colon cancer have higher concentration in proximal colon (8).

Traditionally, two types of genetic instability have been described in CRC: chromosomal instability (CIN) and microsatellite instability (MSI) (9). These tumorigenic pathways have been modeled in two familial cancer syndromes, namely familial adenomatous polyposis (FAP) and hereditary nonpolyposis coli (HNPCC) respectively. Chromosomal instability pathway in colon cancer has been characterized by sequential accumulation of genetic alterations involving activation of oncogenes (like $K-R A S$ ) and inactivation of tumor suppressor genes (like p53) in an adenoma-carcinoma sequence (10). Microsatellite instability in CRC results from defective DNA mismatch repair function associated with either a somatic gene mutation (in familial syndrome) and/or gene methylation (in sporadic forms) (9). Epigenomic instability characterized by $\mathrm{CpG}$ island methylator phenotype (CIMP) has been recently described as an alternative pathway for 
tumorigenesis associated with MSI in sporadic CRCs due to methylation of MLH1 mismatch repair gene (11). CIN- and MSI-associated CRCs show a reversed pattern of distribution along the large intestine. Several studies have shown that both familial and sporadic forms of CIN-associated CRCs tend to occur in distal part of colon while MSI-associated tumors have proximal preference in both familial and sporadic forms $(4,9,12)$. It should be noted, however, that tumor phenotype is only determined by underlying molecular pathway not by anatomical site and that there is significant overlap among proximal and distal CRCs with respect to their underlying molecular mechanism. Reflecting the heterogeneous character of CRCs, recent reports indicate that a subset of CRCs is not associated with either CIN or MSI (13). There is increasing evidence that at least part of CRCs in this group are CIMP positive phenotype associated with lower level of gene methylation and $K-R A S$ mutation (14). Heterogeneity of colon cancer disease has become further evident by an emerging concept that recognizes rectal cancer as a separate entity from the rest of distal colon cancers and tends to divide CRCs into proximal colon cancer, distal colon cancer and rectal cancer (4). Lower frequency of $K-R A S$ mutations, higher level of p53 expression, higher cyclooxygenase-2 (Cox2) and higher nuclear $B$-catenin expression are in support of this distinction (4).

The aim of this study was to perform a comprehensive and simultaneous analysis of clinicopathological, molecular and protein expression profile on a large series of proximal and distal colon carcinomas and rectal cancers in order to provide evidence for the concept of viewing these tumors as distinct clinicopathologic entities based on their anatomic locations.

\section{Patients and methods}

Patients. Primary pre-operatively untreated, unselected sporadic CRC patients $(n=1,420)$ treated at the University Hospital of Basel between the years 1987 and 1996 were included in this study. Hematoxylin and eosin (H\&E) stained slides were retrospectively collected from the Institute of Pathology, University Hospital of Basel, the Institute of Clinical Pathology, Basel, Switzerland and the Institute of Pathology, Stadtspital Triemli, Zürich, Switzerland. Histopathological criteria were reviewed and included tumor diameter, $\mathrm{pT}$ and $\mathrm{pN}$ classification (according to the 6th edition of the AJCC Cancer Staging manual), grade of differentiation, histologic subtype, the presence of vessel invasion, the tumor border configuration (pushing/expanding or infiltrating), as well as the presence of peritumoral lymphocytic inflammation at the invasive tumor front. Clinical data including patient age at diagnosis, gender, and tumor location was reviewed concurrently. Censored observations included patients who were alive at the last follow-up, died for reasons other than $\mathrm{CRC}$ or who were lost to follow-up.

Specimen characteristics. A previously described single-punch tissue microarray was constructed including all 1,420 cases and 57 normal colorectal mucosa samples as controls. Briefly, one tissue cylinder $0.6 \mathrm{~mm}$ in diameter was punched from representative tissue areas and brought into one recipient paraffin block $(3 \times 2.5 \mathrm{~cm})$ using a homemade semi-automated tissue array maker. Of these 1,420 cases, paraffin-embedded surgical resection specimens from 600 cases were available and thus retrospectively collected from the archives of the Institute of Pathology, University Hospital Basel, Switzerland for subsequent molecular analysis.

\section{Assay methods}

Molecular analysis of BRAF, K-RAS and mismatch repair genes. Analysis for $B R A F, K-R A S$ and MSI status are routinely performed at the Institute of Pathology, University Hospital Basel, Switzerland. A detailed protocol has previously been described (15). Microsatellite stable (MSS) and MSI-low (MSI-L) status were defined as instability at 0 and 1 markers, respectively. MSI-H was characterized by the presence of instability in $\geq 2$ markers.

Immunohistochemistry. Immunohistochemistry for the 600 CRCs included on the larger tissue microarrays was performed for 36 tumor-associated protein markers selected to represent the most important signaling pathways in colorectal tumorigenesis (WNT, RAS/MAP kinase, TGF-ß, pAKT signaling) and in processes of tumor progression (angiogenesis, metastasis, apoptosis, proliferation cell cycle, cell adhesion). Immunohistochemistry was also carried out for 14 lymphocyte and inflammatory-associated protein markers chosen to cover the widest possible range of different immune and inflammatory cell types including activated T-cells, regulatory, inducer and helper T-cells, macrophages, monocytes, B-cells and natural killer (NK) cells. A list of protein markers, brief descriptions of function or location, clone, manufacturer and antibody dilution is represented in Table I. Staining was carried out according to the manufacturer's protocol. Negative control tissues underwent the same protocol with the primary antibody omitted.

Evaluation of immunohistochemistry. Lymphocyte/inflammatory-associated protein markers were scored by analyzing the number of positive cells per tissue microarray punch and the total number of cells were classified as negative when 0 positive cells were present, as low, moderate and high when 1-10 positive cells, $11-50$ positive cells and $>50$ positive cells per punch could be observed, respectively. For PD1 and iNOS, cases were scored as the complete absence or presence of any positive cells. For tumor-associated protein markers, the percentage of immunoreactive tumor cells over the total number of tumor cells per punch was scored.

Study design. The study design is outlined in Fig. 1. Briefly, a tissue microarray of 1420 CRCs was constructed. Immunohistochemistry for all 50 markers was performed and staining evaluated. Paraffin-embedded tumor blocks $(n=600)$ for these corresponding patients were available for subsequent DNA extraction. Molecular analysis for $K-R A S, B R A F$, and MSI status as well as information on tumor location was available for 399 patients.

Statistical analysis methods. Differences in tumor location and lymphocyte/inflammatory-associated protein expression were analyzed using the $\chi^{2}$ or Fisher's exact test, where 


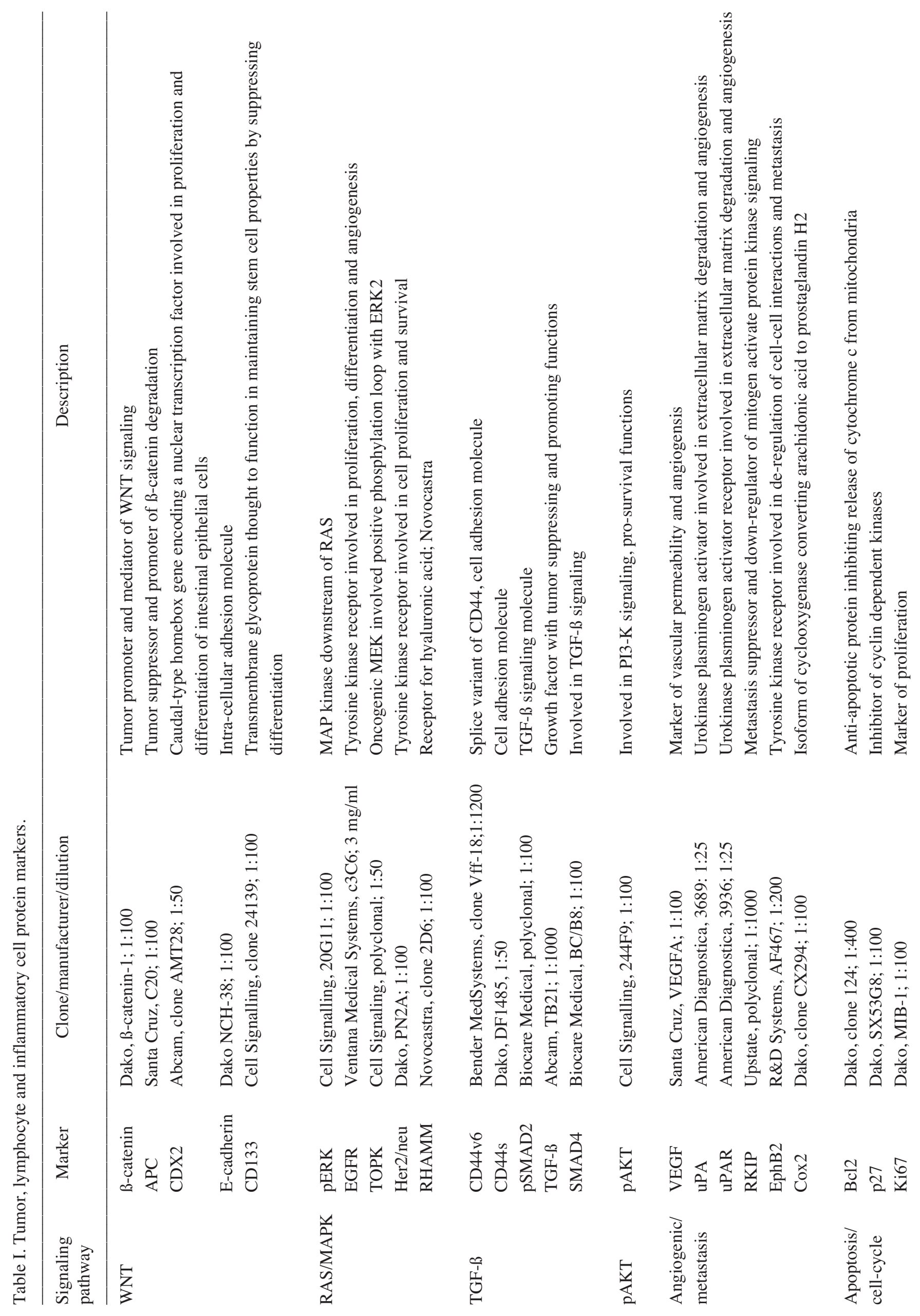




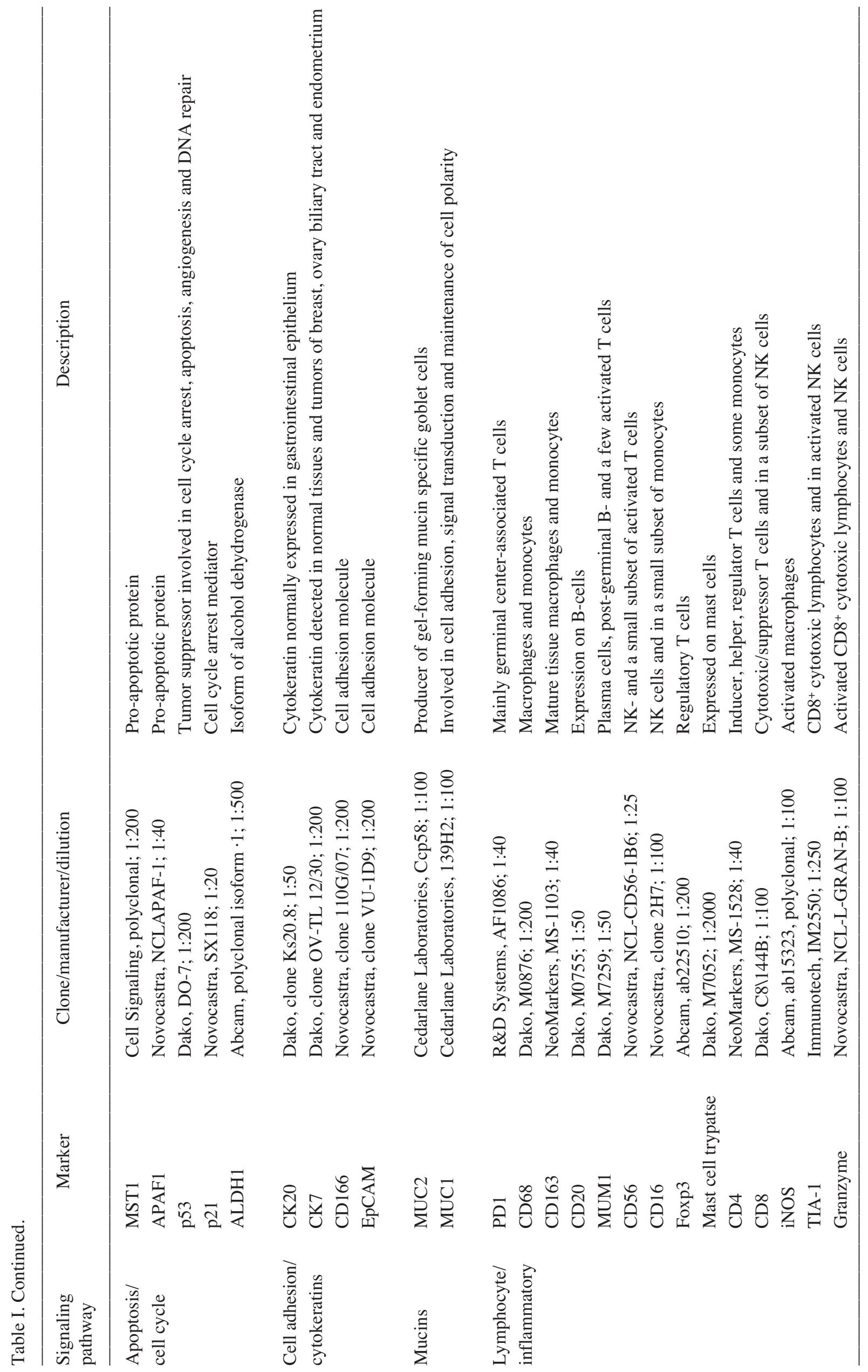




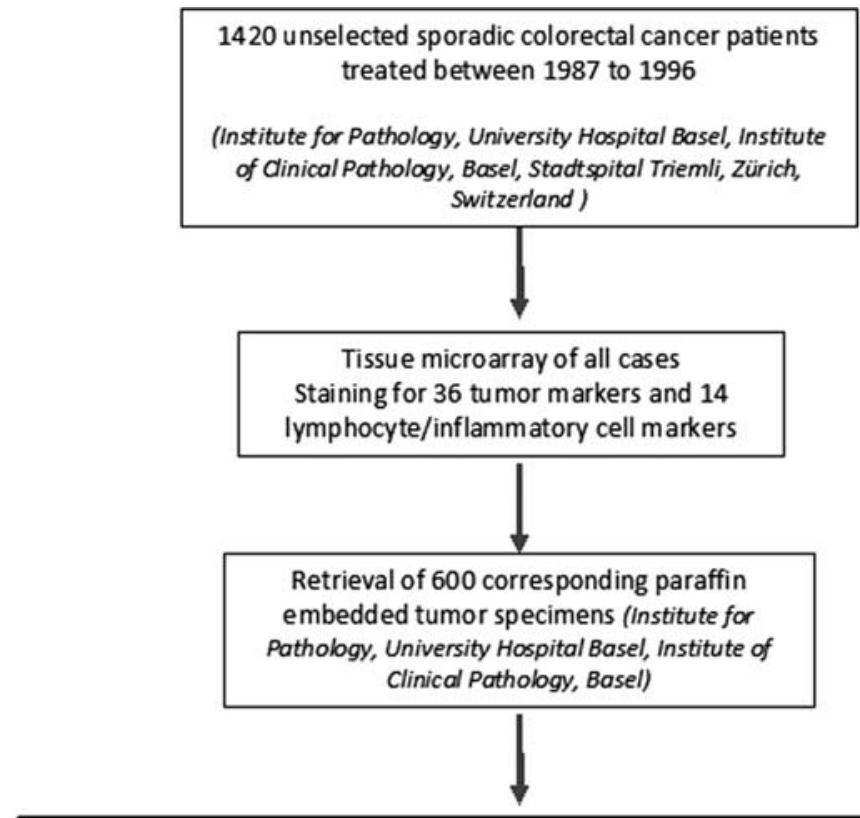

Molecular analysis for : $K$-RAS (exon 2 codon 12/13), BRAF (exon 15, codon V600E) and MSI status: (BAT25, BAT26, D2S123, D5S346, D17S250)

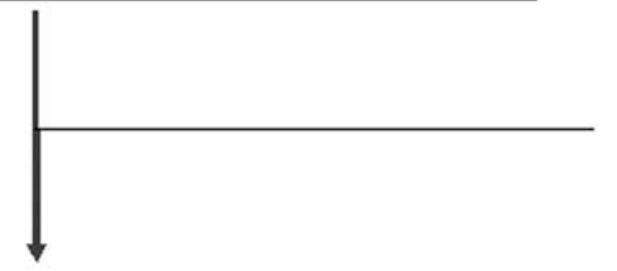

\author{
Exclusion of cases \\ with missing information \\ on tumor location, \\ non-evaluable mutational \\ or $\mathrm{MSI}$ investigations
}

Final number of patients: 399

Figure 1. Study design. Briefly, a tissue microarray of 1420 CRCs was constructed. Immunohistochemistry for all 50 markers was performed and staining evaluated. Six hundred paraffin-embedded tumor blocks for these corresponding patients were available for subsequent DNA extraction. Molecular analysis for $K-R A S, B R A F$, and $M S I$ status as well as information on tumor location was available for 399 patients.

appropriate, whereas age and tumor diameter were analyzed using Student's t-test, and tumor-associated protein expression using the Wilcoxon rank-sum test. In order to determine the discriminatory effect of each variable on tumor location, univariate and multiple logistic regression analysis was performed. Odds ratios and 95\% confidence intervals (CI) were obtained. Receiver operating characteristic (ROC) curve analysis was used to determine the appropriate cut-off scores for all tumor-associated markers and for estimating the discriminatory ability of the final multivariable model for tumor location. Analyses were performed using SAS (V9.1, Cary, NC, USA).

\section{Results}

The total number of patients entered into this study was 399 . Mutational investigations for $K-R A S$ and $B R A F$ were possible in 387 and 369 cases, respectively whereas analysis of MSI status was carried out for all 399 patients. We first screened clinicopathological, molecular and protein expression profile in different anatomical locations of colon and rectum to rule in features/markers that show significant regional difference. We then used these parameters to compare three different regions of colon and rectum in a pair-wise fashion.
Differences in clinicopathological and molecular features stratified by tumor location (Table II). Our initial screening showed multiple clinicopathological/molecular features with significant regional differences. These features include tumor size $(\mathrm{p}<0.001)$, T-stage $(\mathrm{p}<0.001)$, grade $(\mathrm{p}<0.001)$, mucinous histologic type $(\mathrm{p}=0.038), B R A F$ gene mutation (V600E) $(\mathrm{p}=0.002)$ and microsatellite instability $(\mathrm{MSI}-\mathrm{H})(\mathrm{p}=0.002)$. No significant survival time differences were noted between left-sided, right-sided and rectal cancers, although a trend toward worse outcome with right-sided tumor location was observed. Also there was no significant regional difference among CRCs with respect to gender, patient age at the diagnosis, $\mathrm{N}$-stage, vascular invasion, tumor margin type (infiltrating/pushing), peritumoral lymphocytic infiltration and $K$-RAS mutation (codon 12 or 13 ).

Differences in tumor- and immune response-associated protein expression by tumor location (Tables III and IV). We then screened protein expression profiles in specific anatomical regions of colon and rectum which led to the selection of markers showing significant variation in expression among different regions, including CDX2 $(\mathrm{p}<0.001)$, CD44v6 $(\mathrm{p}=0.012)$, CD44s $(\mathrm{p}<0.001)$, TOPK $(\mathrm{p}=0.008)$, nuclear $\beta$ catenin $(p=0.003), p E R K(p=0.042)$, APAF-1 $(p=0.041), E-$ 
Table II. Differences in clinicopathological and molecular features stratified by tumor location (left-sided, right-sided and rectum).

\begin{tabular}{|c|c|c|c|c|}
\hline \multirow[b]{2}{*}{ Clinicopathological features } & \multicolumn{3}{|c|}{ Frequency N (\%) } & \multirow[b]{2}{*}{ P-value } \\
\hline & Left-sided & Rectum & Right-sided & \\
\hline \multicolumn{5}{|l|}{ Gender } \\
\hline Female & $60 \quad(57.7)$ & $80 \quad(50.6)$ & $74 \quad(54.0)$ & 0.53 \\
\hline Male & $44 \quad(42.3)$ & 79 (49.4) & $63(46.0)$ & \\
\hline \multicolumn{5}{|l|}{ Patient age at diagnosis (years) } \\
\hline Mean (min, max) & $68.3(40,95)$ & $69.4(43,90)$ & $70.1(42,93)$ & 0.15 \\
\hline \multicolumn{5}{|l|}{ Tumor diameter (mm) } \\
\hline Mean (min, max) & $47.8(4,120)$ & $44.3(5,100)$ & $57.8(13,170)$ & $<0.001$ \\
\hline \multicolumn{5}{|l|}{ pT stage } \\
\hline pT1-2 & $20(19.6)$ & $50 \quad(32.3)$ & $13 \quad(9.6)$ & $<0.001$ \\
\hline pT3-4 & $82(80.4)$ & $105(67.7)$ & $123(90.4)$ & \\
\hline \multicolumn{5}{|l|}{ pN stage } \\
\hline $\mathrm{pNO}$ & $59(57.8)$ & $76(51.0)$ & $65(48.2)$ & 0.325 \\
\hline $\mathrm{pN} 1-2$ & $43 \quad(42.2)$ & 73 (48.9) & $70 \quad(51.9)$ & \\
\hline \multicolumn{5}{|l|}{ Tumor grade } \\
\hline G1-2 & $100(98.0)$ & $151(87.4)$ & $119(87.5)$ & $<0.001$ \\
\hline G3 & $2 \quad(2.0)$ & $4 \quad(2.6)$ & 17 (12.5) & \\
\hline \multicolumn{5}{|l|}{ Histologic subtype } \\
\hline Mucinous & $8 \quad(7.7)$ & $6 \quad(3.8)$ & $16(11.7)$ & 0.038 \\
\hline Non-mucinous & $96 \quad(92.3)$ & $152(96.2)$ & $121(88.3)$ & \\
\hline \multicolumn{5}{|l|}{ Vascular invasion } \\
\hline Absent & 75 (73.5) & $111(71.6)$ & $88(64.7)$ & 0.275 \\
\hline Present & $27 \quad(26.5)$ & 44 (28.4) & $48 \quad(35.3)$ & \\
\hline \multicolumn{5}{|l|}{ Tumor margin } \\
\hline Infiltrating & $73(72.3)$ & $105(67.7)$ & 97 (71.9) & 0.66 \\
\hline Pushing & $28(27.7)$ & $50 \quad(32.3)$ & $38 \quad(28.2)$ & \\
\hline \multicolumn{5}{|c|}{ Peritumoral lymphocytic infiltration } \\
\hline Absent & 77 (75.5) & $120(77.4)$ & $106(77.9)$ & 0.899 \\
\hline Present & $25 \quad(24.5)$ & 35 (22.6) & $30 \quad(22.1)$ & \\
\hline \multicolumn{5}{|l|}{ Survival rate $(95 \% \mathrm{CI})$} \\
\hline 5-year & $55.5(45-65)$ & $47.6(40-55)$ & $48.8(40-57)$ & 0.327 \\
\hline \multicolumn{5}{|l|}{ KRAS (codon 12 or 13$)$} \\
\hline Wild-type & $77(75.5)$ & $107(69.0)$ & $87(66.9)$ & 0.346 \\
\hline Mutation & $25 \quad(24.5)$ & $48(31.0)$ & $43 \quad(33.1)$ & \\
\hline \multicolumn{5}{|l|}{$B R A F^{V 600 E}$} \\
\hline Wild-type & $86(89.6)$ & $137(93.8)$ & $102(80.3)$ & 0.002 \\
\hline Mutation & $10(10.4)$ & $9 \quad(6.2)$ & $25 \quad(19.7)$ & \\
\hline \multicolumn{5}{|l|}{ Microsatellite instability } \\
\hline MSS/MSI-L & 91 (87.5) & $143(90.5)$ & $104(75.9)$ & 0.002 \\
\hline MSI-H & $13(12.5)$ & $15 \quad(9.5)$ & $33(24.1)$ & \\
\hline
\end{tabular}


Table III. Mean protein expression (\%) and differences by tumor location (left-sided, rectum, right-sided).

\begin{tabular}{|c|c|c|c|c|}
\hline $\begin{array}{l}\text { Protein } \\
\text { markers }\end{array}$ & $\begin{array}{c}\text { Left-sided } \\
(\%)\end{array}$ & $\begin{array}{c}\text { Rectum } \\
(\%)\end{array}$ & $\begin{array}{c}\text { Right-sided } \\
(\%)\end{array}$ & P-value \\
\hline CK20 & 74.4 & 76.1 & 68.9 & 0.118 \\
\hline CK7 & 5.0 & 5.2 & 2.7 & 0.533 \\
\hline CDX2 & 86.7 & 87.7 & 69.6 & $<0.001$ \\
\hline CD44v6 & 51.2 & 63.5 & 66.5 & 0.012 \\
\hline CD44s & 21.5 & 29.2 & 40.4 & $<0.001$ \\
\hline CD133 & 25.2 & 22.1 & 22.9 & 0.402 \\
\hline CD166 & 53.3 & 53.7 & 56.3 & 0.908 \\
\hline EpCAM & 91.1 & 90.7 & 95.9 & 0.198 \\
\hline ALDH1 & 12.0 & 11.5 & 8.4 & 0.622 \\
\hline TOPK & 61.0 & 52.8 & 68.0 & 0.008 \\
\hline Cox2 & 90.8 & 87.2 & 93.4 & 0.231 \\
\hline VEGF & 57.7 & 59.9 & 61.9 & 0.775 \\
\hline ß-catenin & 18.3 & 14.2 & 8.2 & 0.003 \\
\hline Her2/neu & 8.9 & 7.7 & 7.2 & 0.666 \\
\hline EGFR & 58.9 & 61.3 & 68.6 & 0.054 \\
\hline APC & 62.2 & 75.7 & 72.5 & 0.079 \\
\hline pERK & 8.2 & 6.7 & 5.9 & 0.042 \\
\hline $\mathrm{pAKT}$ & 14.9 & 15.9 & 19.5 & 0.123 \\
\hline p53 & 47.9 & 42.8 & 36.8 & 0.261 \\
\hline APAF-1 & 87.8 & 84.8 & 90.3 & 0.041 \\
\hline MST1 & 80.1 & 77.3 & 73.8 & 0.705 \\
\hline E-cadherin & 70.2 & 84.5 & 75.6 & 0.004 \\
\hline RHAMM & 78.0 & 75.1 & 77.1 & 0.525 \\
\hline RKIP & 73.1 & 79.2 & 79.7 & 0.286 \\
\hline pSMAD2 & 55.5 & 50.0 & 52.6 & 0.326 \\
\hline MUC1 & 31.6 & 25.0 & 34.6 & 0.164 \\
\hline MUC2 & 15.6 & 14.2 & 26.3 & 0.06 \\
\hline TGF-ß & 30.1 & 30.2 & 32.4 & 0.581 \\
\hline p21 & 8.2 & 6.5 & 14.0 & 0.003 \\
\hline p27 & 64.1 & 66.9 & 70.9 & 0.104 \\
\hline $\mathrm{Bcl} 2$ & 31.5 & 31.4 & 40.0 & 0.439 \\
\hline EphB2 & 40.6 & 36.9 & 41.4 & 0.571 \\
\hline Ki67 & 21.0 & 24.2 & 27.3 & 0.172 \\
\hline SMAD4 & 21.5 & 20.4 & 23.4 & 0.866 \\
\hline uPAR & 68.6 & 65.9 & 73.3 & 0.088 \\
\hline uPA & 56.0 & 55.6 & 66.8 & 0.005 \\
\hline
\end{tabular}

cadherin $(p=0.004), p 21(p=0.003)$ and uPA $(p=0.005)$. Several immune response associated markers showed significant difference among proximal colon, distal colon and rectum. These include CD68 ( $\mathrm{p}=0.003), \mathrm{CD} 163$ ( $\mathrm{p}=0.013)$, Foxp3 (0.002) and TIA-1 (0.035).

Association of significant predictive parameters with specific tumor locations (Table V). In the next step, we compared predictive parameters among three distinct tumor locations in a pair-wise fashion. When clinicopathological features were compared in different regions, right-sided tumors showed a significantly larger tumor diameter, a more advanced pT stage and a higher tumor grade compared to left-sided tumors ( $\mathrm{p}=0.002,0.012$ and 0.009 respectively) and rectal cancers $(\mathrm{p}<0.001, \mathrm{p}<0.001, \mathrm{p}=0.003$ respectively). The only clinicopathological difference between left-sided colon cancers and rectal cancers was advanced $\mathrm{T}$-stage in the former $(\mathrm{p}=0.01)$. Mucinous histologic feature showed a significant difference only when right-sided tumors were compared with rectal cancers $(\mathrm{p}=0.014)$. With regards to molecular features, MSI$\mathrm{H}$ was significantly more common in proximal colon cancers when compared to distal colon $(\mathrm{p}=0.026)$ and rectal $(\mathrm{p}=0.001)$ cancers. BRAF mutation status was significantly different only if rectal and proximal colon cancers were compared $(\mathrm{p}=0.001)$.

In the tumor marker/immune response marker category, negative expression of CDX2, B-catenin and pERK and positive expression of CD44s, CD44v6, CD68, and CD163 differentiated proximal from distal colon cancers $(\mathrm{p}=0.002$, $\mathrm{p}<0.001, \mathrm{p}=0.021, \mathrm{p}<0.001, \mathrm{p}=0.001, \mathrm{p}<0.001, \mathrm{p}=0.005$, respectively).

Positive expression of TOPK, APAF-1, p21, uPA, Foxp3 and TIA- 1 and negative expression of CDX2 and B-catenin were capable of differentiating right-sided colon from rectal cancers $(\mathrm{p}=0.013,0.008,0.026,0.007,0.002,0.006, \mathrm{p}<0.001$, $\mathrm{p}=0.005$, respectively).

Finally, positive expression of CD44v6, E-cadherin, CD68, CD163 and Foxp3 were found significantly more frequently in rectal cancers compared to left-sided colon cancers $(\mathrm{p}=0.014,0.009,0.047,0.007$ and 0.006$)$.

Most discriminatory factors characterizing distinct tumor locations (Table VI). In order to identify the most discriminating and independent predictive factors for specific tumor locations, significant features/markers in Table $\mathrm{V}$ were reanalyzed using multivariate analysis (Table VI). Independent predictors of proximal colon cancers compared to distal tumors included CD44s positivity $(\mathrm{p}=0.037), \mathrm{CD} 44 \mathrm{v} 6$ positivity $(\mathrm{p}<0.001)$ and negativity for $\beta$-catenin $(\mathrm{p}<0.001)$ as well as higher numbers of CD68 positive cells $(\mathrm{p}=0.017)$. No protein marker was able to best differentiate proximal colon cancers from rectal tumors. However, clinicopathological/ molecular features including higher tumor diameter $(\mathrm{p}=0.005)$, higher T-stage $(\mathrm{p}=0.002)$ and more frequent MSI-H status $(p=0.033)$ were capable of making this distinction. Rectal cancers can be best distinguished from left colon cancers by lower T-stage $(\mathrm{p}=0.004)$ and higher E-cadherin expression $(\mathrm{p}=0.016)($ Table VI).

\section{Discussion}

The results of our study on 399 CRCs show the presence of several differences between cancers originating from proximal colon, distal colon and rectum with respect to their clinicopathological, molecular and protein profiles.

Clinical data analysis revealed that right-sided tumors are significantly larger than tumors in the rest of colon and rectum. Furthermore, T-stage could discriminate right-sided 
Table IV. Lymphocyte/inflammatory marker expression and differences by tumor location (left-sided, rectum, right-sided).

\begin{tabular}{|c|c|c|c|c|}
\hline \multirow[b]{2}{*}{ Protein markers } & \multicolumn{3}{|c|}{ Frequency N (\%) } & \multirow[b]{2}{*}{ P-value } \\
\hline & Left-sided & Rectum & Right-sided & \\
\hline \multicolumn{5}{|l|}{ PD1 } \\
\hline Absent & $101(97.1)$ & $149(94.3)$ & $135(99.3)$ & 0.056 \\
\hline Present & $3(2.9)$ & $9(5.7)$ & $1 \quad(0.7)$ & \\
\hline \multicolumn{5}{|l|}{ CD68 } \\
\hline Negative & $8(7.8)$ & $14(8.9)$ & $5 \quad(3.7)$ & 0.003 \\
\hline Low & $29(28.2)$ & $27(17.2)$ & $25(18.5)$ & \\
\hline Moderate & $46(44.7)$ & $68(43.3)$ & $51(37.8)$ & \\
\hline High & $20(19.4)$ & $48(30.6)$ & $54(40.0)$ & \\
\hline \multicolumn{5}{|l|}{ CD163 } \\
\hline Negative & $5(4.9)$ & $7 \quad(4.5)$ & $5 \quad(3.7)$ & 0.013 \\
\hline Low & $20(19.6)$ & $20(12.9)$ & $18(13.4)$ & \\
\hline Moderate & $47(46.1)$ & $56(36.1)$ & $47(35.1)$ & \\
\hline High & $30(29.4)$ & $72(46.5)$ & $64(47.8)$ & \\
\hline \multicolumn{5}{|l|}{ CD20 } \\
\hline Negative & $59(58.4)$ & $74(48.1)$ & $77(57.0)$ & 0.127 \\
\hline Low & $35(34.7)$ & $58(37.7)$ & $40(29.6)$ & \\
\hline Moderate & 7 (6.9) & $16(10.4)$ & $15(11.1)$ & \\
\hline High & 0 & $6 \quad(3.9)$ & $3(2.2)$ & \\
\hline \multicolumn{5}{|l|}{ MUM1 } \\
\hline Negative & $31(29.8)$ & $36(23.2)$ & $40(29.4)$ & 0.145 \\
\hline Low & $40(38.5)$ & $47(30.3)$ & $41(30.2)$ & \\
\hline Moderate & $22(21.2)$ & $56(36.1)$ & $45(33.1)$ & \\
\hline High & $11(10.6)$ & $16(10.3)$ & $10(7.4)$ & \\
\hline \multicolumn{5}{|l|}{ CD56 } \\
\hline Negative & $86(84.3)$ & $128(82.6)$ & $113(83.7)$ & 0.917 \\
\hline Low & $15(14.7)$ & $23(14.8)$ & $18(13.3)$ & \\
\hline Moderate & $1(1.0)$ & 4 (2.6) & $4(3.0)$ & \\
\hline \multicolumn{5}{|l|}{ CD16 } \\
\hline Negative & $2(1.9)$ & 3 (1.9) & $1 \quad(0.8)$ & 0.9 \\
\hline Low & $21(20.8)$ & $33(21.0)$ & $33(24.6)$ & \\
\hline Moderate & $59(58.4)$ & $85(54.1)$ & $69(51.5)$ & \\
\hline High & $19(18.8)$ & $36(22.9)$ & $31(23.1)$ & \\
\hline \multicolumn{5}{|l|}{ Foxp3 } \\
\hline Negative & $34(33.0)$ & $27(17.2)$ & $31(23.0)$ & 0.002 \\
\hline Low & $30(29.1)$ & $43(27.4)$ & $54(40.0)$ & \\
\hline Moderate & 37 (35.9) & $80(51.0)$ & $45(33.3)$ & \\
\hline High & 2 (1.9) & 7 (4.5) & $5 \quad(3.7)$ & \\
\hline \multicolumn{5}{|l|}{ Mast cells } \\
\hline Negative & $12(11.7)$ & $18(11.5)$ & $17(12.7)$ & 0.242 \\
\hline Low & $52(50.5)$ & $63(40.1)$ & $63(47.0)$ & \\
\hline Moderate & $36(35.0)$ & $67(42.7)$ & $49(36.6)$ & \\
\hline High & 3 (2.9) & $9(5.7)$ & $5 \quad(3.7)$ & \\
\hline
\end{tabular}


Table IV. Continued.

\begin{tabular}{|c|c|c|c|c|}
\hline \multirow[b]{2}{*}{ Protein markers } & \multicolumn{3}{|c|}{ Frequency N (\%) } & \multirow[b]{2}{*}{ P-value } \\
\hline & Left-sided & Rectum & Right-sided & \\
\hline \multicolumn{5}{|l|}{ CD4 } \\
\hline Negative & $62(63.3)$ & $94(62.7)$ & $76(61.3)$ & 0.742 \\
\hline Low & $33(33.7)$ & $43(28.7)$ & $33(26.6)$ & \\
\hline Moderate & $2(2.0)$ & $13 \quad(8.7)$ & $14(11.3)$ & \\
\hline High & $1(1.0)$ & $\begin{array}{ll}0 & (0.0)\end{array}$ & $1 \quad(0.8)$ & \\
\hline \multicolumn{5}{|l|}{ CD8 } \\
\hline Negative & $30(13.3)$ & $48(31.4)$ & $45(34.4)$ & 0.695 \\
\hline Low & $53(55.2)$ & $77(50.3)$ & $53(40.5)$ & \\
\hline Moderate & $12(12.5)$ & $27(17.7)$ & $25(19.1)$ & \\
\hline High & $1 \quad(1.0)$ & $1 \quad(0.7)$ & $8 \quad(6.1)$ & \\
\hline \multicolumn{5}{|l|}{ iNOS } \\
\hline Absent & $56(65.7)$ & 95 (64.6) & $94(75.8)$ & 0.107 \\
\hline Present & $34(34.3)$ & $52(35.4)$ & $30(24.2)$ & \\
\hline \multicolumn{5}{|l|}{ TIA-1 } \\
\hline Negative & $53(54.1)$ & $88(62.0)$ & $54(45.0)$ & 0.035 \\
\hline Low & 39 (39.8) & $44(31.0)$ & $56(46.7)$ & \\
\hline Moderate & $6(6.1)$ & $10 \quad(7.0)$ & $8(6.7)$ & \\
\hline High & $\begin{array}{ll}0 & (0.0)\end{array}$ & $\begin{array}{ll}0 & (0.0)\end{array}$ & $2(1.7)$ & \\
\hline \multicolumn{5}{|l|}{ Granzyme } \\
\hline Negative & $35(35.4)$ & $47(32.2)$ & $41(32.3)$ & 0.312 \\
\hline Low & $55(56.0)$ & $79(54.1)$ & $59(46.5)$ & \\
\hline Moderate & $8 \quad(8.1)$ & $17(11.6)$ & $24(18.9)$ & \\
\hline High & $1 \quad(1.0)$ & $3(2.1)$ & $3(2.4)$ & \\
\hline
\end{tabular}

cancers from left-sided colon and rectal cancers (Table V) with rectal cancers showing the lowest T-stage. Interestingly, in the multivariate analysis, T-stage showed independent predictive value for discriminating rectal cancers from proximal and distal colon cancers (Table VI). Some studies have suggested a direct association between increasing age, female sex and proximal location in colon cancers (16). However, our data, in line with some other studies $(17,18)$, do not support this association (Table II). We found that proximal cancers exhibit significantly higher tumor grade as compared to distal colon $(\mathrm{p}=0.009)$ and rectal cancers $(\mathrm{p}=0.003)$ (Table V). This is in agreement with two previous reports $(12,16)$, whereas other studies did not show any regional difference in tumor grade (17). Furthermore, mucinous tumors were found more frequently in the right side only when it was compared to rectum ( $\mathrm{p}=0.014)$ (Table $\mathrm{V})$. This finding is also in agreement with some $(5,12,16,17)$ but not all previous reports $(19,20)$. Survival was not affected by tumor location in our hands (Table II) contrary to studies reporting a better survival for proximal colon cancers $(8,12)$ and a worse survival for rectal tumors (21).
Our results were also significant for a regional difference in CRC genetic profile. In accordance to most previous reports, our data indicate that MSI-H status is more common in proximal colon cancers as compared to distal colon $(\mathrm{p}=0.026)$ and rectum $(\mathrm{p}=0.001)$ (Table V) $(5,6,12,17)$. However, $B R A F$ mutation showed a significant difference only when rightsided and rectal cancers were compared (Table V). Using molecular data, MSI-H status was able to independently predict proximal colon location in the multivariate analysis only when proximal colon cancers were compared to rectal cancers (Table VI). No significant regional difference was identified for $K-R A S$ mutation which is contrary to prior reports showing more frequent $K-R A S$ mutation in proximal colon and less frequency in rectum $(22,23) . K-R A S$ mutation has recently been recognized as the best predictor of CIMP-low or CIMP2 CRCs that are characterized by lacking both MSI and CIN (Introduction) and not showing any regional preference $(13,14)$. Our data from regional $K-R A S$ mutation analysis are in support of this concept.

We also examined regional differences in expression of tumor marker proteins as well as immune response markers 
Table V. Univariate analysis of protein expression, clinicopathological and molecular features with specific tumor locations: right vs left-sided, right-sided versus rectal and rectal versus left-sided tumors. ${ }^{a}$

\begin{tabular}{|c|c|c|c|c|c|c|c|}
\hline \multirow[b]{2}{*}{ Feature } & \multirow[b]{2}{*}{$\begin{array}{l}\text { Cut-off score for } \\
\text { protein markers }\end{array}$} & \multicolumn{2}{|c|}{ Right vs. left } & \multicolumn{2}{|c|}{ Right vs. rectum } & \multicolumn{2}{|c|}{ Rectum vs. left } \\
\hline & & OR $(95 \% \mathrm{CI})$ & P-value & OR $(95 \% \mathrm{CI})$ & $\mathrm{P}$-value & OR $(95 \%$ CI) & P-value \\
\hline Diameter & & $1.02(1.01-1.03)$ & 0.002 & $1.03(1.02-1.04)$ & $<0.001$ & $0.99(0.9-1.0)$ & NS \\
\hline pT stage & & $1.68(1.12-2.52)$ & 0.012 & $3.46 \quad(2.2-5.5)$ & $<0.001$ & $0.61(0.4-0.9)$ & 0.01 \\
\hline Tumor grade & & $7.14 \quad(1.6-31.7)$ & 0.009 & $5.39 \quad(1.8-16.5)$ & 0.003 & $1.32(0.2-7.4)$ & NS \\
\hline $\begin{array}{l}\text { Histologic } \\
\text { subtype }\end{array}$ & & 1.59 (0.6-3.9) & NS & $3.35 \quad(1.3-8.8)$ & 0.014 & $0.47(0.2-1.4)$ & NS \\
\hline $\begin{array}{l}B R A F^{V 600 E} \\
\text { mutation }\end{array}$ & & $2.11 \quad(1.0-4.6)$ & NS & $3.73(1.7-8.4)$ & 0.001 & $0.57(0.2-1.4)$ & NS \\
\hline MSI status & & $2.21 \quad(1.1-4.5)$ & 0.026 & $3.02 \quad(1.6-5.9)$ & 0.001 & $0.73(0.3-1.6)$ & NS \\
\hline $\mathrm{CDX} 2$ & $95 \%$ & $0.42 \quad(0.3-0.7)$ & 0.002 & $0.35 \quad(0.2-0.6)$ & $<0.001$ & $1.22(0.7-2.1)$ & NS \\
\hline CD44v6 & $30 \%$ & $2.54 \quad(1.4-4.5)$ & 0.001 & $1.28 \quad(0.7-2.2)$ & 0.374 & $1.98(1.2-3.4)$ & 0.014 \\
\hline $\mathrm{CD} 44 \mathrm{~s}$ & $35 \%$ & $2.79(1.5-5.1)$ & $<0.001$ & $1.63(0.9-2.7)$ & NS & $1.71(0.9-3.1)$ & NS \\
\hline TOPK & $95 \%$ & $1.52(0.8-2.8)$ & NS & $2.04 \quad(1.2-3.6)$ & 0.013 & $0.75(0.4-1.4)$ & NS \\
\hline B-catenin & $30 \%$ & $0.15(0.06-0.4)$ & $<0.001$ & $0.26 \quad(0.1-0.7)$ & 0.005 & $0.59(0.3-1.1)$ & NS \\
\hline pERK & $5 \%$ & $0.47 \quad(0.2-0.9)$ & 0.021 & $(0.4-1.5)$ & NS & $0.59(0.3-1.1)$ & NS \\
\hline APAF-1 & $95 \%$ & $1.45(0.8-2.5)$ & NS & $1.96(1.2-3.2)$ & 0.008 & $0.74(0.4-1.3)$ & NS \\
\hline E-cadherin & $95 \%$ & $1.86(0.9-3.7)$ & NS & $0.75 \quad(0.4-1.5)$ & NS & $2.49(1.3-4.9)$ & 0.009 \\
\hline p21 & $15 \%$ & $1.59(0.8-3.2)$ & NS & $2.12(1.1-4.1)$ & 0.026 & $0.75(0.4-1.6)$ & NS \\
\hline uPA & $60 \%$ & $1.7 \quad(1.0-3.1)$ & NS & $2.06 \quad(1.2-3.5)$ & 0.007 & $0.83(0.5-1.5)$ & NS \\
\hline CD68 & 50 cells/punch & $2.77 \quad(1.5-5.0)$ & $<0.001$ & $1.51 \quad(0.9-2.5)$ & NS & $1.83(1.1-3.3)$ & 0.047 \\
\hline CD163 & 50 cells/punch & $2.19(1.3-3.8)$ & 0.005 & 1.05 (0.6-1.7) & NS & $2.08(1.3-3.5)$ & 0.007 \\
\hline Foxp3 & 10 cells/punch & $0.97 \quad(0.6-1.6)$ & NS & $0.47 \quad(0.3-0.8)$ & 0.002 & $2.04(1.3-3.4)$ & 0.006 \\
\hline TIA-1 & 0 cells/punch & $1.39(0.5-3.9)$ & NS & $1.99(1.2-3.3)$ & 0.006 & $1.16(0.4-3.3)$ & NS \\
\hline
\end{tabular}

aOR, odds ratio. CI, confidence interval. NS, not significant.

Table VI. Most discriminatory factors characterizing specific tumor locations in multivariable analysis.

\begin{tabular}{lllllllll}
\hline Right vs. left & OR $(95 \% \mathrm{CI})$ & P-value & Right vs. rectum & OR $(95 \% \mathrm{CI})$ & P-value & Rectum vs. left & OR (95\% CI) & P-value \\
\hline CD44s & $2.28(1.1-5.0)$ & 0.037 & Tumor diameter & $1.03(1.0-1.1)$ & 0.005 & pT stage & $0.52(0.3-0.8)$ & 0.004 \\
CD44v6 & $2.27(1.06-4.9)$ & 0.034 & pT stage & $2.85(1.5-5.6)$ & 0.002 & E-cadherin & $2.38(1.2-4.8)$ & 0.016 \\
B-catenin & $0.14(0.05-0.4)$ & $<0.001$ & MSI status & $3.01(1.1-8.3)$ & 0.033 & & & \\
CD68 & $2.45(1.2-5.1)$ & 0.017 & & & & & & \\
\hline
\end{tabular}

OR, odds ratio. $\mathrm{CI}$, confidence interval.

(Tables III-V). CDX2, CD44s, TOPK, nuclear ß-catenin, pERK, APAF-1, E-cadherin, p21 and uPA were the only tumor markers and CD68, CD163, Foxp3 and TIA-1 were the only immune response markers that showed significant regional 
A

Right versus left:

$\uparrow$ CD44s
$\uparrow$ CD44v6
$\downarrow$ B-catenin
$\uparrow$ CD68+

B

Right versus rectum:

$\uparrow$ Tumor diameter
$\uparrow$ pT stage
$\uparrow$ MSI-H

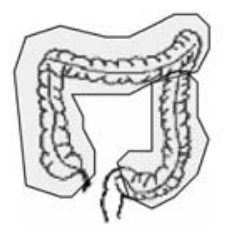

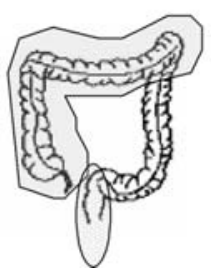

C

Rectum versus left:

$\uparrow \mathrm{E}$-cadherin

$\uparrow$ p T stage

Figure 2. Representation of independent predictive factors discriminating between specific tumor locations. Tumors located on the right side of the colon differed from left-sided colon cancers in terms of over-expression of CD44s, CD44v6 and CD68 and decreased expression of B-catenin. Differences in rightsided tumors compared to rectum included a larger tumor diameter, more advance pT stage and a greater frequency of MSI-H on the right side. Comparing rectum to left-sided colon cancers, rectal tumors showed increased expression of E-cadherin and a more advanced pT stage.

differences (Tables III and IV). These markers were used to compare three CRC locations in pairs to specify regions with significantly higher or lower expressions. Based on this analysis, proximal colon cancers can be differentiated from distal colon cancers by negative CDX2, and nuclear ß-catenin expression, higher CD44s expression and more frequent CD68 and CD163 positive cells (Table V). Loss of CDX2 expression in right-sided tumors was independent of MSIstatus confirming previous reports $(24,25)$. When these results were adjusted for multiple testing, only CDX2 expression loss and CD44 overexpression were selected as discriminator between right- and left-sided colon cancers $\left(\mathrm{p}<0.001\right.$, both). In clinical practice a panel of $\mathrm{CDX} 2^{+} \mathrm{CK} 7^{-}$ and $\mathrm{CK} 20^{+}$has been traditionally employed to confirm a colorectal cancer origin in the case of metastasis of unknown primary (26). This is, however, inconsistent with the finding of CDX2 expression loss in subset of colon cancers especially those occur in right side. Therefore, loss of CDX2 expression should be interpreted with caution for diagnostic purposes.

Our data show that CD44s and CD44v6 expression has an independent predictive value in differentiating proximal colon from distal colon cancers in a multivariate analysis (Table VI). In addition, further analysis showed a direct association between CD44s expression and MSI-high status $(\mathrm{p}=0.031) . \mathrm{CD} 44$ has recently emerged as a stem cell marker $(27,28)$. These results may suggest a distinct, CD44s-positive stem cell for MSI-high colon cancers, however, CD133 and CD166, the other stem cell markers, did not show any significant regional difference in expression (Table III). Peritumoral macrophage infiltration could correctly classify proximal from distal tumors evidenced by a higher CD68 expression. Marked infiltration of tumor by lymphocyte is shown to be associated with MSI-high status (29), however, we did not detect any significant regional difference in CD8positive tumor infiltrating lymphocytes (TILs) (Table IV). Negative nuclear $\beta$-catenin was another independent predictive marker capable of differentiating proximal colon from distal colon cancers in a multivariate analysis (Table VI) which is consistent with previous data associating left-sided colon cancers with chromosomal instability and APC/catenin pathway (Introduction).

Proximal colon tumors can be distinguished from rectal tumors by negative CDX2 and $B$-catenin expression, higher TOPK, APAF-1, p21 and uPA expression and less frequent Foxp3 positive cells (Table V). We found that p21 is significantly more expressed in right-sided colon cancers compared to rectal cancers in contrast to previous reports (30) showing no regional difference. Also we did not detect any significant difference in regional expressions of p27 and P53 contrary to previous studies that show higher P53 expression in distal colon and rectum $(5,12,31)$ and a lower expression of p27 in proximal colon tumors $(17,32)$. Cox 2 is reported to be more expressed in distal colon or rectal cancers $(4,33)$ however, we did not detect any significant regional Cox 2 expression (Table III). Multivariate analysis showed that higher tumor diameter, higher T-stage and MSI-H status are the best discriminatory factors to differentiate proximal colon cancers from rectal tumors (Table VI and Fig. 2).

Tumors located in the rectum demonstrated more similarity to tumors located in distal colon in terms of clinicopathological, molecular and protein expression profile. Higher E-cadherin expression and more frequent CD68, CD163 and Foxp3 positive cells were the only markers that were able to differentiate rectal cancers from the tumors in distal colon (Table V). In multivariate analysis, lower T-stage and higher E-cadherin expression were the best independent predictors in differentiating rectal cancers from left-sided colon cancers (Fig. 2).

Taken together, our data indicate the presence of significant regional differences in CRCs with respect to their clinicopathological, molecular pathogenesis and protein profile. The distinction is more prominent when proximal colon cancers are compared with distal colon and rectal cancers and less dramatic in comparison between distal colon and rectal 
cancers. These data along with existing evidence for the presence of distinct regional embryological origin and gene expression profile are highly supportive of the concept that proximal and distal CRCs are distinct clinicopathologic entities. This concept has practical implications in prevention and treatment of both familial and sporadic CRCs.

\section{Acknowledgements}

Dr Parham Minoo is a recipient of CAP Foundation Resident Research Grant supported by unrestricted grants from Abbott Molecular, Cleveland Clinic Foundation, McKesson Provider Technologies, Sakura Finetek USA, and Sysmex USA.

\section{References}

1. Jemal A, Siegel R, Ward E, Hao Y, Xu J and Thun MJ: Cancer statistics, 2009. CA Cancer J Clin 59: 225-249, 2009.

2. Cucino C, Buchner AM and Sonnenberg A: Continued rightward shift of colorectal cancer. Dis Colon Rectum 45: 1035-1040, 2002.

3. Rabeneck L, Davila JA and El-Serag HB: Is there a true 'shift' to the right colon in the incidence of colorectal cancer? Am J Gastroenterol 98: 1400-1409, 2003.

4. Li FY and Lai MD: Colorectal cancer, one entity or three. J Zhejiang Univ Sci B 10: 219-229, 2009.

5. Iacopetta B: Are there two sides to colorectal cancer? Int J Cancer 101: 403-408, 2002.

6. Bufill JA: Colorectal cancer: evidence for distinct genetic categories based on proximal or distal tumor location. Ann Intern Med 113: 779-788, 1990.

7. Glebov OK, Rodriguez LM, Nakahara K, et al: Distinguishing right from left colon by the pattern of gene expression. Cancer Epidemiol Biomarkers Prev 12: 755-762, 2003.

8. Gervaz P, Bucher P and Morel P: Two colons-two cancers: paradigm shift and clinical implications. J Surg Oncol 88: 261-266, 2004

9. Jass JR: Molecular heterogeneity of colorectal cancer: implications for cancer control. Surg Oncol 16: S7-S9, 2007.

10. Vogelstein B, Fearon ER, Hamilton SR, et al: Genetic alterations during colorectal-tumor development. N Engl J Med 319: $525-532,1988$

11. Herman JG, Umar A, Polyak K, et al: Incidence and functional consequences of hMLH1 promoter hypermethylation in colorectal carcinoma. Proc Natl Acad Sci USA 95: 6870-6875, 1998.

12. Gervaz P, Bouzourene H, Cerottini JP, et al: Dukes B colorectal cancer: distinct genetic categories and clinical outcome based on proximal or distal tumor location. Dis Colon Rectum 44: 364-372, 2001.

13. Issa JP: Colon cancer: it's CIN or CIMP. Clin Cancer Res 14: 5939-5940, 2008.

14. Shen L, Toyota M, Kondo Y, et al: Integrated genetic and epigenetic analysis identifies three different subclasses of colon cancer. Proc Natl Acad Sci USA 104: 18654-18659, 2007.

15. Zlobec I, Baker K, Terracciano L, et al: Two-marker protein profile predicts poor prognosis in patients with early rectal cancer. Br J Cancer 99: 1712-1717, 2008.
16. Nawa T, Kato J, Kawamoto H, et al: Differences between rightand left-sided colon cancer in patient characteristics, cancer morphology and histology. J Gastroenterol Hepatol 23: 418-423, 2008.

17. Azzoni C, Bottarelli L, Campanini N, et al: Distinct molecular patterns based on proximal and distal sporadic colorectal cancer: arguments for different mechanisms in the tumorigenesis. Int $\mathbf{J}$ Colorectal Dis 22: 115-126, 2007.

18. Mensink PB, Kolkman JJ, van Baarlen J and Kleibeuker JH: Change in anatomic distribution and incidence of colorectal carcinoma over a period of 15 years: clinical considerations. Dis Colon Rectum 45: 1393-1396, 2002.

19. Green JB, Timmcke AE, Mitchell WT, Hicks TC, Gathright JB Jr and Ray JE: Mucinous carcinoma - just another colon cancer? Dis Colon Rectum 36: 49-54, 1993.

20. Secco GB, Fardelli R, Campora E, et al: Primary mucinous adenocarcinomas and signet-ring cell carcinomas of colon and rectum. Oncology 51: 30-34, 1994.

21. Kalady MF, Sanchez JA, Manilich E, Hammel J, Casey G and Church JM: Divergent oncogenic changes influence survival differences between colon and rectal adenocarcinomas. Dis Colon Rectum 52: 1039-1045, 2009.

22. Frattini M, Balestra D, Suardi S, et al: Different genetic features associated with colon and rectal carcinogenesis. Clin Cancer Res 10: 4015-4021, 2004.

23. Slattery ML, Curtin K, Wolff RK, et al: A comparison of colon and rectal somatic DNA alterations. Dis Colon Rectum 52: 1304-1311, 2009

24. Baba Y, Nosho K, Shima K, et al: Relationship of CDX2 loss with molecular features and prognosis in colorectal cancer. Clin Cancer Res 15: 4665-4673, 2009.

25. Okon K, Zazula M, Rudzki Z, Papla B, Osuch C and Stachura J: CDX-2 expression is reduced in colorectal carcinomas with solid growth pattern and proximal location, but is largely independent of MSI status. Pol J Pathol 55: 9-14, 2004.

26. Lugli A, Tzankov A, Zlobec I and Terracciano LM: Differential diagnostic and functional role of the multi-marker phenotype CDX2/CK20/CK7 in colorectal cancer stratified by mismatch repair status. Mod Pathol 21: 1403-1412, 2008.

27. Dalerba P, Dylla SJ, Park IK, et al: Phenotypic characterization of human colorectal cancer stem cells. Proc Natl Acad Sci USA 104: 10158-10163, 2007.

28. Chu P, Clanton DJ, Snipas TS, et al: Characterization of a subpopulation of colon cancer cells with stem cell-like properties. Int J Cancer 124: 1312-1321, 2009.

29. Jass JR, Whitehall VL, Young J and Leggett BA: Emerging concepts in colorectal neoplasia. Gastroenterology 123: 862-876, 2002.

30. Lam AK, Ong K and Ho YH: hTERT expression in colorectal adenocarcinoma: correlations with p21, p53 expressions and clinicopathological features. Int J Colorectal Dis 23: 587-594, 2008.

31. Kapiteijn E, Liefers GJ, Los LC, et al: Mechanisms of oncogenesis in colon versus rectal cancer. J Pathol 195: 171-178, 2001.

32. Zhang H and Sun XF: Loss of p27 expression predicts poor prognosis in patients with Dukes' B stage or proximal colorectal cancer. Int J Oncol 19: 49-52, 2001.

33. Birkenkamp-Demtroder K, Olesen SH, Sorensen FB, et al: Differential gene expression in colon cancer of the caecum versus the sigmoid and rectosigmoid. Gut 54: 374-384, 2005. 